\title{
Serum interleukin 6 and acute urinary retention in elderly men with benign prostatic hyperplasia in China: a cross-sectional study
}

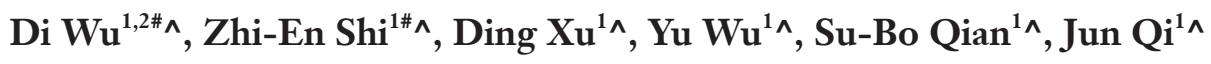 \\ ${ }^{1}$ Department of Urology, Xinhua Hospital Affiliated to Shanghai Jiao Tong University School of Medicine, Shanghai, China; ${ }^{2}$ Department of \\ Urology, Huadong Hospital Affiliated to Fudan University, Shanghai, China \\ Contributions: (I) Conception and design: D Wu, J Qi; (II) Administrative support: D Xu, J Qi; (III) Provision of study materials or patients: D Wu, \\ ZE Shi; (IV) Collection and assembly of data: D Wu, ZE Shi; (V) Data analysis and interpretation: D Xu, Y Wu, SB Qian; (VI) Manuscript writing: \\ All authors; (VII) Final approval of manuscript: All authors. \\ \#These authors contributed equally to this work. \\ Correspondence to: Jun Qi. Department of Urology, Xinhua Hospital Affiliated to Shanghai Jiao Tong University School of Medicine, Shanghai 20092, \\ China. Email: qijun@xinhuamed.com.cn.
}

Background: While acute urinary retention (AUR) is a severe complication of benign prostatic hyperplasia $(\mathrm{BPH})$, it can also indicate the progression of this common disease in aging men. This study aimed at exploring the possible relationship between serum interleukin 6 (sIL-6) and AUR in BPH patients.

Methods: A cross-sectional study was conducted based on 256 elderly men with BPH in China. The association between the sIL-6 level and the occurrence of AUR was evaluated by univariate and multivariable logistic regressions. The receiver operating characteristic (ROC) curve was utilized to determine the discriminant validity of the sIL-6 level and the optimal cut-off value.

Results: The concentration of sIL-6 was significantly elevated in the AUR group $(\mathrm{P}<0.001)$. A positive correlation was observed between the sIL-6 level and AUR in BPH patients [odds ratio $(\mathrm{OR})=1.365,95 \%$ confidence interval (CI): 1.174-1.586, $\mathrm{P}<0.001$ ]. Based on the ROC curve analysis for sIL-6, the optimal cutoff point of $4.475 \mathrm{pg} / \mathrm{mL}$ was set to identify the occurrence of AUR in these patients [area under the curve $($ AUC $)=0.7596,95 \%$ CI: 0.6976-0.8216, P<0.001]. A high sIL-6 level $(\geq 4.475 \mathrm{pg} / \mathrm{mL})$ had a significantly stronger correlation with AUR (OR =9.666, 95\% CI: 4.347-21.491, P<0.001).

Conclusions: There was a positive correlation between the sIL-6 level and the occurrence of AUR in elderly Chinese patients with BPH. This study provides potential strategies for the screening of BPH individuals with a possible risk of AUR, which may contribute to the early implementation of effective interventions to improve the quality of life and prognosis. Long-term prospective studies are still required to further illustrate the causal relationship.

Keywords: Interleukin-6; prostatic hyperplasia; serum; urinary retention

Submitted May 08, 2020. Accepted for publication Nov 06, 2020.

doi: 10.21037/tau-20-914

View this article at: http://dx.doi.org/10.21037/tau-20-914

^ ORCID: Di Wu, 0000-0003-3129-1640; Zhi-En Shi, 0000-0002-6192-7366; Ding Xu, 0000-0002-4086-7819; Yu Wu, 0000-0001-80558327; Su-Bo Qian, 0000-0001-6097-6484; Jun Qi, 0000-0003-0970-8462. 


\section{Introduction}

Benign prostatic hyperplasia (BPH) occurs mostly in men older than 50 years old. Chronically progressive, $\mathrm{BPH}$ is the most common benign disease that causes dysuria in older men. Repeated urinary tract infections, stones, hematuria, acute urinary retention (AUR), and renal dysfunction are clinical manifestations of the progression of BPH (1-3). As one of the most severe complications of $\mathrm{BPH}$, the estimated prevalence rate of AUR in elderly men with BPH has been reported to reach to about 53\% (4). Moreover, AUR has been one of the most common reasons for surgical treatment. AUR suggests the presence of a decompensation of bladder function, and bladder dysfunction directly affects patients' prognosis $(5,6)$. An increasing number of studies have shown that, compared with patients without a history of AUR, the efficacy of surgery for BPH patients with a history of AUR is significantly worse (7). Early intervention in patients with a known AUR risk can avoid irreversible damage to the bladder function and can help to improve the treatment efficacy of BPH. Therefore, the discovery of indicators to more accurately predict the risk of AUR in patients with $\mathrm{BPH}$ is essential and of great clinical significance.

Growing evidence has shown that inflammation plays a vital role in the occurrence and progression of $\mathrm{BPH}(8-10)$. Chronic inflammation can be found in most $\mathrm{BPH}$ pathological specimens (11-13); thus, BPH has been regarded as an immune inflammatory disease (14-16). Relevant studies have confirmed that prostatic inflammation is strongly correlated with AUR in BPH patients (17-22). Moreover, the severity of the inflammatory response is positively associated with both the occurrence of AUR and the requirement for surgical operations (23). Inflammatory mediators are essential to the entire process of both occurrence and development of systemic or local inflammation, and they have been reported to be involved in prostatic inflammation, which results in the progression of BPH, including AUR (10). Therefore, certain inflammatory mediators may help to predict the possible risk of AUR in $\mathrm{BPH}$ patients. Moreover, our previous study has found that the serum level of interleukin (IL)-8 is positively associated with the probability of suffering AUR in elderly men with BPH (24).

Similar to prostate-specific antigen (PSA), IL-6 has been used to evaluate prostatic inflammation and cancer (25-29), but no report exists concerning the use of serum IL-6 (sIL-6) to evaluate the risk of AUR in elderly men with BPH. This cross-sectional study provides possible evidence for sIL-6 as a promising biomarker for predicting the potential risk of AUR in BPH patients. We analyzed the relationship between sIL-6 levels and the occurrence of AUR in these patients, intending to gain insight into the optimal treatment efficacy and the improvement of prognosis and quality of life. We present this research in accordance with the STrengthening the Reporting of OBservational studies in Epidemiology (STROBE) reporting checklist (available at http://dx.doi.org/10.21037/tau-20-914).

\section{Methods}

\section{Study population and design}

We conducted a cross-sectional study of patients diagnosed with $\mathrm{BPH}$ at the first visit to the Department of Urology in Xinhua Hospital (Shanghai, China) from $1^{\text {st }}$ January 2017 to $30^{\text {th }}$ November 2019. Based on the presence of AUR at recruitment, participants were allocated into either the AUR group or the AUR-free group, and sIL-6 was also measured at this time. Patients with a history of lower urinary tract injury or surgery, medical treatment of BPH including $\alpha$-adrenergic blockers and/or $5 \alpha$-reductase inhibitors in the past 1 year, urogenital malignancy, or any other comorbidities involving the voiding dysfunction, such as neurogenic bladder dysfunction, severe urinary tract infection, bladder stones, and psychological diseases, were excluded. The necessary sample size of this study was calculated using the following formula: $\mathrm{N} \geq 50+8 \mathrm{~m}(\mathrm{~N}=$ the sample size; $\mathrm{m}=$ the number of independent variables) (30-32), which indicated that the sample size needed to be $\geq 242$ to enable multivariable regression analysis. Finally, a sample of 256 participants was recruited for this study. A series of measures, including the systemic training for researchers, rigorous plan for data collection, and stringent quality control, were taken to minimize selection bias, information bias, and other biases.

The baseline data, including age, body mass index (BMI), and comorbidities, such as diabetes mellitus (DM), cardiovascular disease (CVD), hypertension, and neuropathy, were systematically evaluated for each patient. Laboratory examinations involving levels of total protein (TP), albumin (Alb), creatinine (Cr), uric acid (UA), total cholesterol (TC), low-density lipoprotein cholesterol (LDL-c), high-density lipoprotein cholesterol (HDL-c), 
triglycerides (TG), plasma glucose (Glu), blood white blood cell (WBC) counts, and urine WBC counts were performed in accordance with the technical laboratory standards of our hospital. The blood concentrations of PSA and total testosterone (TT) were detected by electrochemiluminescence immunoassay (ECLIA). An enzyme-linked immunosorbent assay (ELISA) and turbidimetric inhibition immunoassay (TINIA) were used to determine the sIL-6 level and C-reactive protein (CRP) concentration, respectively. Prostatic anatomical factors were assessed by transrectal ultrasound (TRUS) using a Siemens ultrasound machine (Sequoia 512, Siemens Medical Solutions, Mountain View, CA, USA) with a transrectal probe (EV8C4-S). A Laborie UDS64III urodynamic system (Laborie Medical Technologies, Brossard, QC, Canada) was utilized to record the maximum flow rate $(\mathrm{Qmax})$ and detrusor pressure at maximum flow rate (PdetQmax).

AUR was defined as a sudden inability to urinate voluntarily (33) that required catheterization to void retained urine. The BMI $\left(\mathrm{kg} / \mathrm{m}^{2}\right)$ was calculated as the body weight $(\mathrm{kg})$ divided by the square of height $(\mathrm{m})$. The total prostate volume (TPV, $\mathrm{mL}$ ) was calculated based on the following formula: the maximum length of anteroposterior dimension $(\mathrm{L}, \mathrm{cm}) \times$ the maximum width of transverse dimension $(\mathrm{W}, \mathrm{cm}) \times$ the maximum height of superoinferior dimension $(\mathrm{H}, \mathrm{cm}) \times 0.52$ (34). The transitional zone volume $(\mathrm{TZV}, \mathrm{mL})$ of the prostate was calculated in the same way. The transitional zone index (TZI) was calculated as the TZV (mL) divided by the TPV (mL) (35). The vertical distance between the top of the mid-lobe on the sagittal plane and the base of the bladder neck was utilized to describe the intravesical prostatic protrusion (IPP, mm) (36). The density of PSA (PSAD) was defined as the PSA $(\mathrm{ng} / \mathrm{mL})$ divided by the TPV $(\mathrm{mL})$. The lower urinary tract symptoms (LUTS) were evaluated by the International Prostate Symptom Score (I-PSS). A prostate biopsy was applied to patients with abnormal digital rectal examination findings or elevated PSA levels ( $\geq 4 \mathrm{ng} / \mathrm{mL}$ ), while patients diagnosed with prostate cancer via the biopsy were excluded.

This study was conducted in accordance with the Declaration of Helsinki (as revised in 2013). The study protocol was approved by the Ethics Committee in Xinhua Hospital Affiliated to Shanghai Jiao Tong University School of Medicine (XHEC-D-2020-078). All participants provided written informed consent.

\section{Statistical analysis}

Quantitative data were described as the mean \pm standard deviation (SD) or the median (interquartile range). The distribution's normality was verified by a one-sample Kolmogorov-Smirnov test. The independent-samples $t$-test was performed if the data accorded with a normal distribution. The Mann-Whitney $U$ test was used when the data did not accord with a normal distribution. Qualitative data were expressed as frequencies (\%). Intergroup differences were analyzed by the chi-squared $\left(\chi^{2}\right)$ test or the Fisher's exact test. The associations between AUR and relevant factors of interest were evaluated by univariate and multivariable logistic regressions via the enter and forward likelihood ratio (FLR) method, respectively. The baseline variables that indicated a univariate relationship with the occurrence of AUR were further analyzed by the multivariable logistic regressions. The discriminant validity of sIL- 6 was evaluated by the receiver operating characteristic (ROC) curve and the area under the curve (AUC), whereas Youden's index was applied to obtain the optimal cutoff value. Analyses were conducted by SPSS Statistics (version 25.0, IBM Corp., Armonk, NY, USA), and the ROC curve was plotted by R software (version 3.5.1, R Foundation for Statistical Computing, Vienna, Austria). A two-sided $\mathrm{P}$ value of $<0.05$ was considered to indicate statistical significance.

\section{Results}

A total of $256 \mathrm{BPH}$ patients were recruited in this study (Figure 1). The median age was 71 (interquartile range, 66-77) years old, and the prevalence of AUR was $33.20 \%$ $(85 / 256)$. The demographic and clinical characteristics of the overall study population are displayed in Table 1.

The univariate analysis was performed by univariate logistic regression (Table 2). Total PSA (t-PSA), free PSA (f-PSA), f/t-PSA, PSAD, TZI, Qmax, PdetQmax, sIL-6, Alb, and TG were all correlated with AUR $(\mathrm{P}<0.05)$.

Based on the results of the multivariable logistic regression analysis, sIL-6 was positively correlated with AUR after a series of adjustments. Table 3 showed that compared with the results of univariate analyses, the multivariable logistic regression results were also significant $[\beta=0.220$, odds ratio $(\mathrm{OR})=1.246,95 \%$ confidence interval (CI): $1.141-1.362, \mathrm{P}<0.001]$ by adjusting for Alb and TG. After further adjusting for PSA-related variables and TZI, the significance remained $(\beta=0.293, \mathrm{OR}=1.340,95 \%$ 


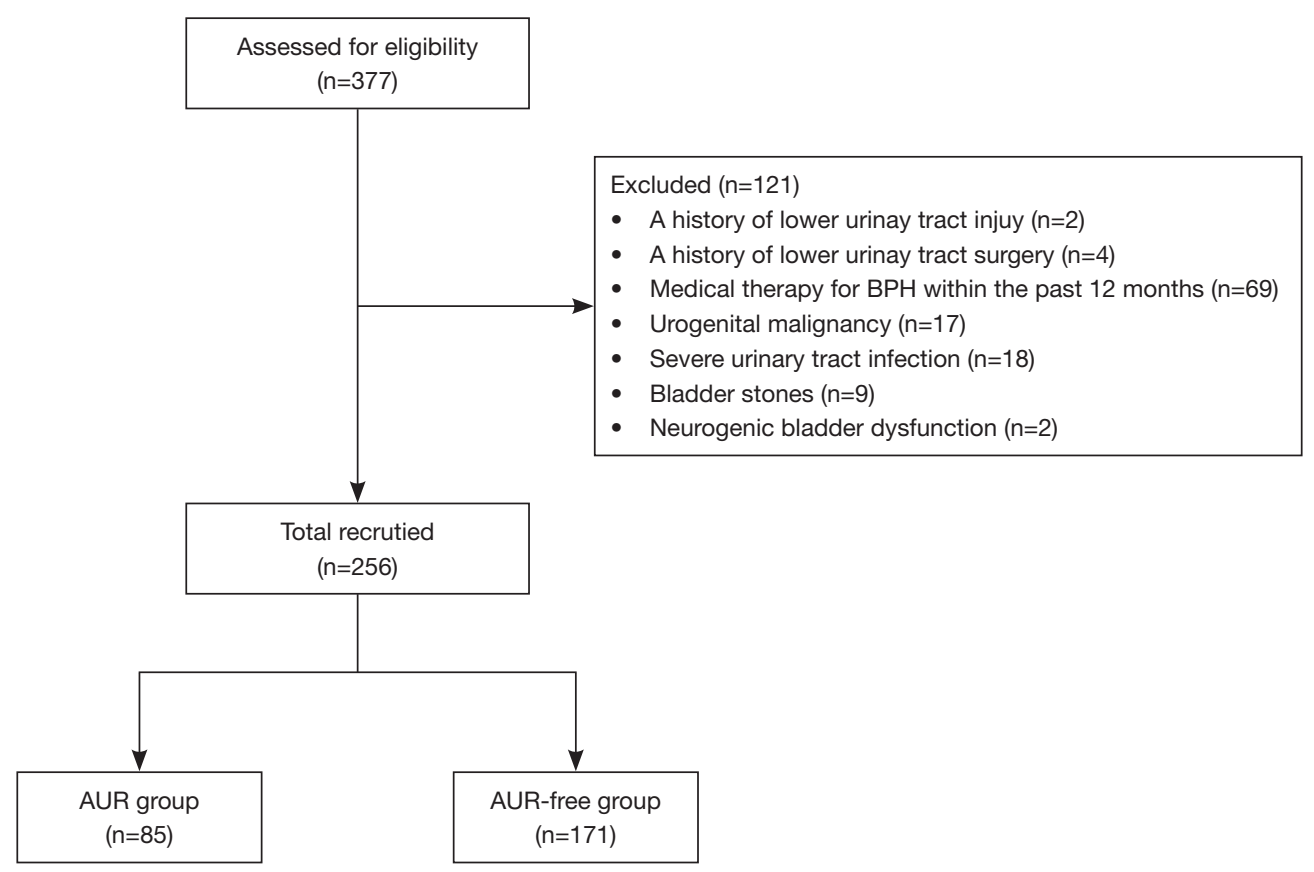

Figure 1 The flow diagram of recruitment in this study. AUR, acute urinary retention; BPH, benign prostatic hyperplasia.

Table 1 The baseline data of enrolled participants in both groups

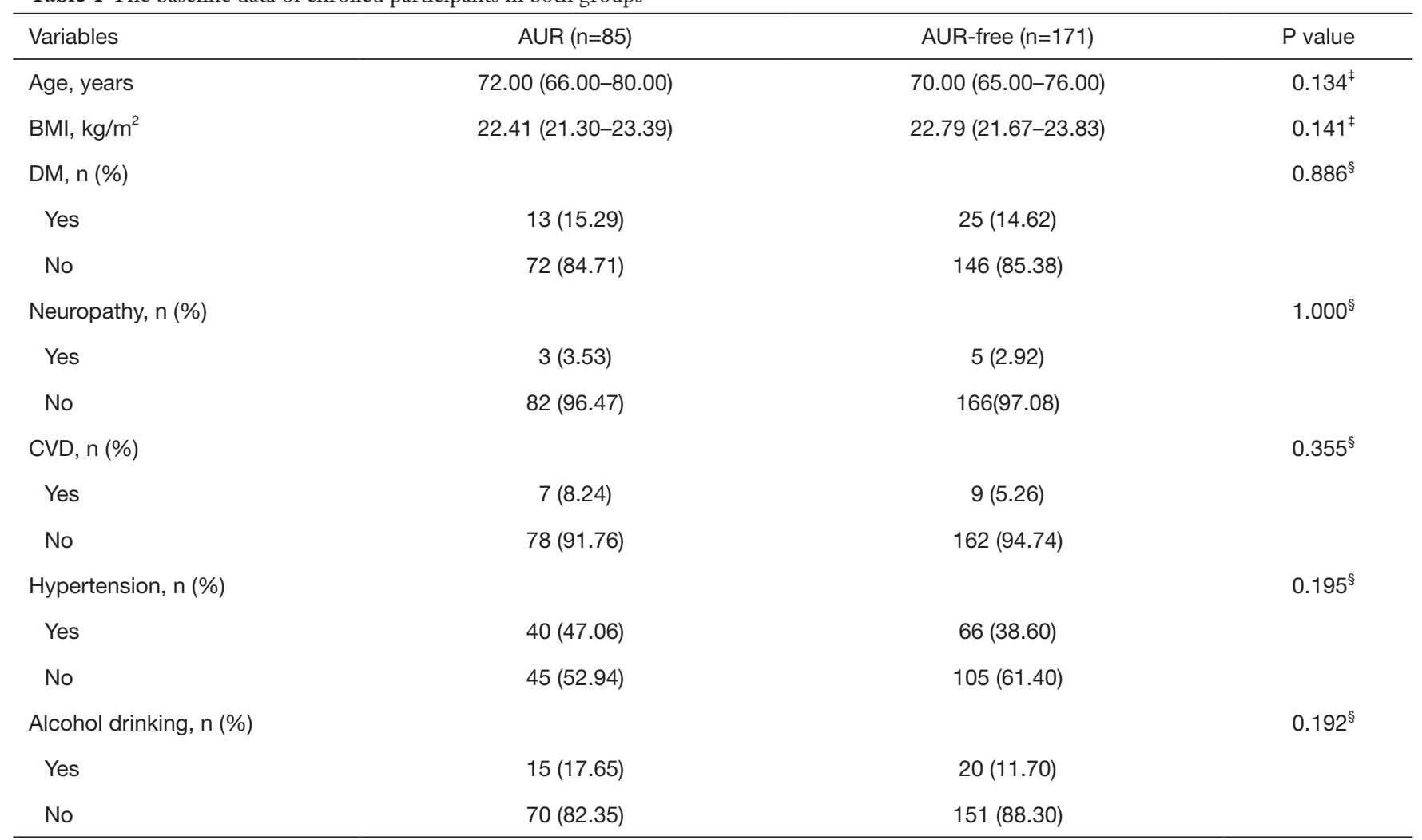

Table 1 (continued) 
Table 1 (continued)

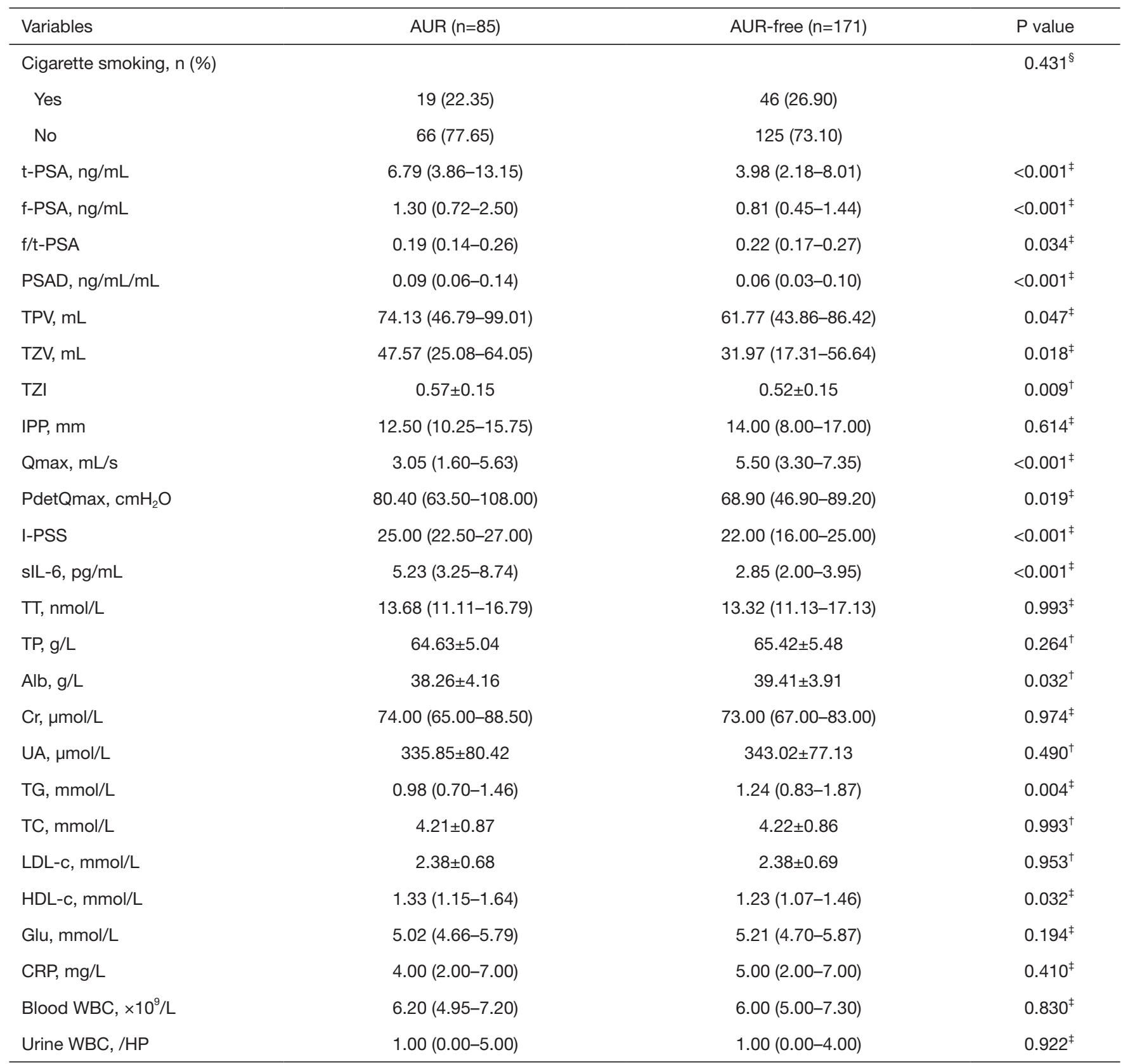

Data are presented as the mean \pm standard deviation (SD), the median (interquartile range), or the frequency (\%). ${ }^{\dagger}$, Student's $t$-test; $\ddagger$, Mann-Whitney U test; ${ }^{\S}$, Chi-squared test or Fisher's exact test. AUR, acute urinary retention; BMI, body mass index; DM, diabetes mellitus; CVD, cardiovascular disease; t-PSA, total prostate-specific antigen; f-PSA, free prostate-specific antigen; f/t-PSA, free to total prostate-specific antigen ratio; PSAD, prostate-specific antigen density; TPV, total prostate volume; TZV, transitional zone volume; TZI, transitional zone index; IPP, intravesical prostatic protrusion; Qmax, maximum flow rate, PdetQmax, detrusor pressure at maximum flow rate; I-PSS, International Prostate Symptom Score; sIL-6, serum interleukin 6; TT, total testosterone; TP, total protein; Alb, albumin; Cr, creatinine; UA, uric acid; TG, triglycerides; TC, total cholesterol; LDL-c, low-density lipoprotein cholesterol; HDL-c, high-density lipoprotein cholesterol; Glu, plasma glucose; CRP, C-reactive protein; WBC, white blood cell. 
Table 2 Associations between relevant factors and the acute urinary retention in elderly men with benign prostatic hyperplasia based on univariate analyses

\begin{tabular}{|c|c|c|c|}
\hline Parameter & Odds ratio & $95 \% \mathrm{Cl}$ & $P$ value \\
\hline Age, years & 1.022 & $0.990-1.054$ & 0.175 \\
\hline BMI, $\mathrm{kg} / \mathrm{m}^{2}$ & 0.975 & $0.868-1.095$ & 0.668 \\
\hline DM, yes/no & 1.054 & $0.510-2.182$ & 0.886 \\
\hline Neuropathy, yes/no & 1.215 & $0.283-5.207$ & 0.793 \\
\hline CVD, yes/no & 1.615 & $0.580-4.498$ & 0.359 \\
\hline $\begin{array}{l}\text { Hypertension, } \\
\text { yes/no }\end{array}$ & 1.414 & $0.836-2.392$ & 0.196 \\
\hline $\begin{array}{l}\text { Alcohol drinking, } \\
\text { yes/no }\end{array}$ & 1.618 & $0.782-3.347$ & 0.195 \\
\hline $\begin{array}{l}\text { Cigarette smoking, } \\
\text { yes/no }\end{array}$ & 0.782 & $0.424-1.443$ & 0.432 \\
\hline t-PSA, ng/mL & 1.055 & $1.020-1.092$ & 0.002 \\
\hline f-PSA, ng/mL & 1.240 & $1.044-1.472$ & 0.014 \\
\hline f/t-PSA & 0.030 & $0.001-0.951$ & 0.047 \\
\hline $\mathrm{PSAD}, \mathrm{ng} / \mathrm{mL} / \mathrm{mL}$ & 40.511 & $2.662-616.445$ & 0.008 \\
\hline TPV, mL & 1.004 & $0.997-1.010$ & 0.241 \\
\hline $\mathrm{TZV}, \mathrm{mL}$ & 1.006 & $0.998-1.013$ & 0.161 \\
\hline TZI & 11.449 & $1.797-72.949$ & 0.010 \\
\hline IPP, mm & 0.967 & $0.911-1.027$ & 0.273 \\
\hline Qmax, mL/s & 0.806 & $0.712-0.912$ & 0.001 \\
\hline$P \operatorname{det} Q \max , \mathrm{cmH}_{2} \mathrm{O}$ & 1.012 & $1.002-1.022$ & 0.014 \\
\hline slL-6, pg/mL & 1.194 & $1.108-1.287$ & $<0.001$ \\
\hline$\pi$, nmol/L & 0.986 & $0.933-1.043$ & 0.628 \\
\hline TP, g/L & 0.972 & $0.925-1.021$ & 0.263 \\
\hline Alb, g/L & 0.931 & $0.871-0.995$ & 0.034 \\
\hline $\mathrm{Cr}, \mu \mathrm{mol} / \mathrm{L}$ & 1.002 & $0.992-1.012$ & 0.648 \\
\hline $\mathrm{UA}, \mu \mathrm{mol} / \mathrm{L}$ & 0.999 & $0.995-1.002$ & 0.489 \\
\hline $\mathrm{TG}, \mathrm{mmol} / \mathrm{L}$ & 0.607 & $0.411-0.896$ & 0.012 \\
\hline $\mathrm{TC}, \mathrm{mmol} / \mathrm{L}$ & 0.999 & $0.736-1.354$ & 0.993 \\
\hline LDL-c, mmol/L & 0.988 & $0.672-1.454$ & 0.952 \\
\hline HDL-c, mmol/L & 2.157 & $0.958-4.854$ & 0.063 \\
\hline Glu, mmol/L & 0.947 & $0.789-1.136$ & 0.557 \\
\hline
\end{tabular}

Table 2 (continued)
Table 2 (continued)

\begin{tabular}{lccc}
\hline Parameter & Odds ratio & $95 \% \mathrm{Cl}$ & P value \\
\hline CRP, mg/L & 0.998 & $0.957-1.040$ & 0.917 \\
Blood WBC, $\times 10^{9} / \mathrm{L}$ & 1.020 & $0.876-1.187$ & 0.797 \\
Urine WBC, /HP & 1.003 & $0.988-1.017$ & 0.703 \\
\hline
\end{tabular}

BMI, body mass index; DM, diabetes mellitus; CVD, cardiovascular disease; t-PSA, total prostate-specific antigen; f-PSA, free prostate-specific antigen; f/t-PSA, free to total prostate-specific antigen ratio; PSAD, prostate-specific antigen density; TPV, total prostate volume; TZV, transitional zone volume; TZI, transitional zone index; IPP, intravesical prostatic protrusion; Qmax, maximum flow rate, PdetQmax, detrusor pressure at maximum flow rate; sIL-6, serum interleukin 6; TT, total testosterone; TP, total protein; Alb, albumin; $\mathrm{Cr}$, creatinine; UA, uric acid; TG, triglycerides; TC, total cholesterol; LDL-C, low-density lipoprotein cholesterol; HDL-c, high-density lipoprotein cholesterol; Glu, plasma glucose; CRP, C-reactive protein; WBC, white blood cell.

Table 3 The multivariable logistic regression analyses of the serum interleukin 6 level and the acute urinary retention in elderly men with benign prostatic hyperplasia

\begin{tabular}{lcccc}
\hline Assessment & Beta & Odds ratio & $95 \% \mathrm{Cl}$ & P value \\
\hline Model 1 $^{\dagger}$ & 0.220 & 1.246 & $1.141-1.362$ & $<0.001$ \\
${\text { Model } 2^{\ddagger}}$ & 0.293 & 1.340 & $1.190-1.509$ & $<0.001$ \\
Model 3 & 0.311 & 1.365 & $1.174-1.586$ & $<0.001$ \\
\hline${ }^{\dagger}$, Model 1: adjusted for albumin (Alb) and triglycerides (TG); ${ }^{\ddagger}$, \\
Model 2: based on Model 1, further adjusted for total prostate- \\
specific antigen (t-PSA), free prostate-specific antigen (f-PSA), \\
free to total prostate-specific antigen ratio (f/t-PSA), prostate- \\
specific antigen density (PSAD), and transitional zone index \\
(TZI); ${ }^{\S}$, Model 3: based on Model 2, additionally adjusted for \\
maximum flow rate (Qmax) and detrusor pressure at maximum \\
flow rate (PdetQmax).
\end{tabular}

CI: $1.190-1.509, \mathrm{P}<0.001)$. By adjusting for additional univariates involving Qmax and PedtQmax, the ultimate results still indicated the statistical significance $(\beta=0.311$, $\mathrm{OR}=1.365,95 \%$ CI: $1.174-1.586, \mathrm{P}<0.001)$.

According to the ROC analysis for sIL-6, the sIL6 level of $4.475 \mathrm{pg} / \mathrm{mL}$ was the optimal cutoff point for discriminating the occurrence of AUR from the $\mathrm{BPH}$ patients, with a sensitivity of $63.53 \%$ and a specificity of 81.29\% (AUC $=0.7596,95 \%$ CI: $0.6976-0.8216, \mathrm{P}<0.001$ ) (Figure 2). Then, similar univariate and multivariable 


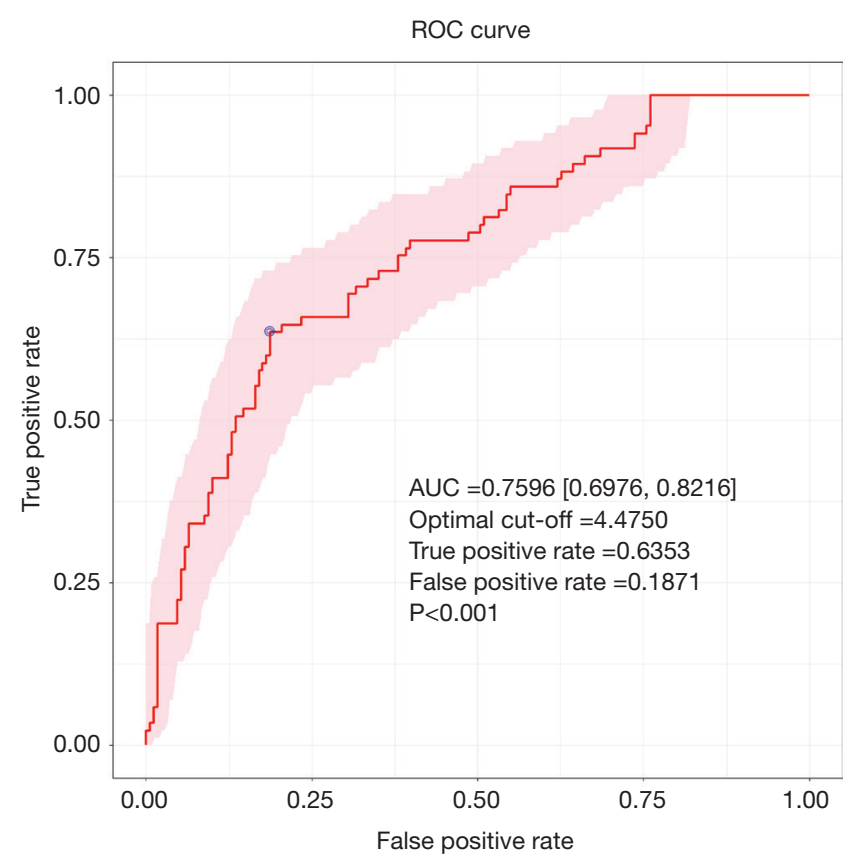

Figure 2 The receiver operating characteristic (ROC) curve for the serum interleukin 6 (sIL-6) level in discriminating the occurrence of acute urinary retention (AUR) in elderly men with benign prostatic hyperplasia (BPH). AUC, area under the curve.

Table 4 The multivariable logistic regression analyses of the high serum interleukin 6 level $(\geq 4.475 \mathrm{pg} / \mathrm{mL})$ and the acute urinary retention in elderly men with benign prostatic hyperplasia

\begin{tabular}{lcccc}
\hline Assessment & Beta & Odds ratio & $95 \% \mathrm{Cl}$ & P value \\
\hline $\begin{array}{l}\text { Univariate } \\
\text { analysis }\end{array}$ & 2.024 & 7.567 & $4.214-13.588$ & $<0.001$ \\
${\text { Model } 1^{\dagger}}^{\dagger}$ & 2.095 & 8.127 & $4.473-14.763$ & $<0.001$ \\
Model 2 $^{\ddagger}$ & 2.103 & 8.188 & $4.315-15.537$ & $<0.001$ \\
Model 3 $^{\S}$ & 2.269 & 9.666 & $4.347-21.491$ & $<0.001$ \\
\hline
\end{tabular}

${ }^{\dagger}$, Model 1: adjusted for albumin (Alb) and triglycerides (TG); ${ }^{\ddagger}$, Model 2: based on Model 1, further adjusted for total prostatespecific antigen (t-PSA), free prostate-specific antigen (f-PSA), free to total prostate-specific antigen ratio ( $f / t-P S A)$, prostatespecific antigen density (PSAD), and transitional zone index (TZI); ${ }^{\S}$, Model 3: based on Model 2, additionally adjusted for maximum flow rate (Qmax) and detrusor pressure at maximum flow rate (PdetQmax).

logistic regression analyses were performed. As shown in Table 4, high levels of sIL-6 correlated with AUR more significantly in all the above models $(\beta=2.095,2.103$, and 2.269, $\mathrm{OR}=8.127,8.188$, and 9.666, $\mathrm{P}<0.001$ ).

\section{Discussion}

The occurrence of AUR, as one of the most serious complications and the critical progressive manifestations of $\mathrm{BPH}$, indicates that the patient has developed the decompensation of the bladder function based on bladder outlet obstruction (6). Although surgical treatment can alleviate some degree of the obstruction, it remains difficult for patients to fully recover bladder function. If the patient can receive timely active treatment before AUR occurs, bladder function can be protected to ensure that the progress of BPH can be effectively delayed and the prognosis can be improved (5). Therefore, an ideal predictor of AUR will help in the development of more reasonable and effective treatment strategies. At present, it is widely believed that prostatic inflammation is involved in both occurrence and progression of BPH (8-10). As mentioned above, inflammatory mediators have been reported to be associated with prostatic inflammation (10). Consequently, some prostatic inflammation-related inflammatory mediators may contribute to the progression of $\mathrm{BPH}$, especially resulting in the occurrence of AUR. The positive association between the serum interleukin 8 concentration and the risk of AUR in elderly men suffered BPH has been revealed by our previous study (24). Moreover, high levels of sIL-6 have been confirmed as correlating with an increased risk of BPH in a dose-dependent pattern (37). Moreover, prostatic inflammation can induce prostate stromal cells to secrete IL-6, leading to the proliferation of prostatic tissues via the IL-17-dependent $\mathrm{BPH}$-associated inflammatory processes (38). This study found that the sIL-6 level independently correlated with the occurrence of AUR in patients with BPH, which might lay a possible foundation for using sIL- 6 as a potential biomarker for predicting the occurrent risk of AUR in elderly men with $\mathrm{BPH}$.

There are many risk factors for AUR, including age, urinary flow rate, prostatitis, IPP, prostate volume, PSA, and detrusor contractility (39-41). In particular, the relationship between prostatitis and AUR has received widespread attention from urologists. Research by Nickel et al. found that prostatic inflammation was present in approximately $77.6 \%$ of prostate biopsy specimens (12). Gerstenbluth et al. observed specimens from 40 prostatectomies and reported that multifocal chronic inflammation could be found in $87.5 \%$ of transitional zone specimens and $95 \%$ of peripheral zone specimens (11). Bao et al. showed that prostatic inflammation was positively correlated with the occurrence 
of AUR in patients with $\mathrm{BPH}$, and the occurrent risk of AUR in BPH patients with prostatic inflammation was 2.362 times that of the patients without relevant inflammatory manifestations $(\mathrm{P}<0.05)(42)$. Limited by the invasive nature of assessment, histological evidence from prostatic inflammation is not suitable for serving as a monitoring indicator for the AUR risk evaluation. Currently, the level of inflammatory mediators in body fluids is used as a clinical marker to assess the inflammatory status and can be obtained through noninvasive methods. Therefore, inflammatory mediators are more suitable for regular monitoring than histological examination and can be used as surrogate alternative indicators to a certain extent.

The cytokine IL-6 plays a crucial role in the acute phase of inflammation and governs the transition from the acute to the chronic stage of inflammation (43). Elevated sIL-6 levels have been reported to be associated with a variety of chronic diseases, including inflammatory prostatitis $(43,44)$. Currently, IL-6 has been regarded as one of the best surrogate markers of chronic inflammation in prostate cancer (26), but there is little research on the relationship between IL-6 and BPH-related inflammation. Penna et al. studied a series of seminal plasma cytokines and chemokines in prostatic inflammation and BPH and found that IL-6 concentration in seminal plasma could not effectively discriminate between chronic prostatitis/chronic pelvic pain syndrome (CP/CPPS) and healthy controls (45). However, Korrovits et al. revealed that the IL- 6 concentration in expressed prostatic secretions (EPSs) could identify prostatitis from healthy controls with a sensitivity and specificity of $80 \%$ and $73 \%$, respectively, at a cutoff value of $50 \mathrm{nmol} / \mathrm{L}$ (25). Compared with seminal plasma and EPSs, blood is more convenient to obtain, and local irritation to prostatic inflammation can be avoided to the greatest extent. At present, no study has explored the relationship between sIL-6 and AUR in patients with BPH. Our study was the first to explore this potential link and obtained relatively satisfactory results. We confirmed that the sIL-6 level was independently correlated with the occurrence of AUR in patients with BPH. The optimal sIL-6 cutoff of $4.475 \mathrm{pg} / \mathrm{mL}$ could effectively identify the occurrence of AUR in BPH patients with a sensitivity of $63.53 \%$ and a specificity of $81.29 \%$ (AUC $=0.7596, \mathrm{P}<0.001$ ). Furthermore, after a series of adjustments for relevant variables, compared with the sIL-6 at a low level $(<4.475 \mathrm{pg} / \mathrm{mL})$, the association between a high level of sIL-6 ( $\geq 4.475 \mathrm{pg} / \mathrm{mL})$ and AUR in elderly men with BPH was more significant $(\mathrm{P}<0.001)$. All these results indicate a strong positive correlation between the concentration of sIL-6 and the AUR risk in aging men with BPH. Moreover, this study might provide some support for the use of sIL-6 as a noninvasive biomarker to evaluate the probability of suffering AUR in patients with $\mathrm{BPH}$, which is conducive to optimizing treatment protocols and improving the prognosis and the quality of life.

The diagnosis and treatment of $\mathrm{BPH}$ are focused on the early prediction of the occurrence of AUR to enable more appropriate treatment opportunities. Inflammation is generally involved in the occurrence and progression of $\mathrm{BPH}$, but the causal relationship between inflammatory mediators and AUR may be the key issue of further study. Another critical direction of study in this field might be the combined detection of multiple inflammatory mediators to enable more accurate predictive abilities of the risk of AUR in BPH patients. Furthermore, we expect the comprehensive assessment of inflammatory mediators obtained via different approaches, including both the body fluid samples (blood and urine) and tissue specimens, to further enhance the reliability of predicting the occurrent risk of AUR in patients with BPH. Moreover, detecting inflammasomes and inflammatory exosomes may also be a critical breakthrough in indicating the risk of suffering AUR in $\mathrm{BPH}$ patients.

Nevertheless, our study had some limitations. The present study was cross-sectional research with a small sample size based on the experience of a single center and was performed over a short time; all these properties might have affected the reliability of the results. Furthermore, the present study focused on only IL-6, and the possible interactions with other inflammatory mediators might have thus been neglected. In addition, the sIL-6 levels detected after the occurrence of AUR might not be equivalent to the actual concentrations before suffering AUR. Despite the statistical significance, the study cannot be used to definitively confirm a causal relationship. Therefore, the results regarding the relationship between sIL-6 and AUR in BPH patients should still be interpreted with caution. In order to verify the validity of sIL-6 as an available biomarker in predicting the potential risk of AUR in elderly men with BPH, a relevant prospective cohort study and basic research have been planned to further confirm the causal relationship and explore related mechanisms.

\section{Conclusions}

The present study found that the sIL-6 level was positively 
correlated with the occurrence of AUR in elderly men with $\mathrm{BPH}$ in China. After adjusting for the relevant AUR risk factors, the sIL-6 at a level of $\geq 4.475 \mathrm{pg} / \mathrm{mL}$ remained significantly associated with the occurrence of AUR. This study might provide support for sIL-6 as a potential predictive biomarker to screen $\mathrm{BPH}$ individuals at risk of AUR, which may contribute to the early implementation of effective medication or surgical interventions to improve the quality of life and prognosis by preventing or delaying the occurrence of bladder decompensation. However, additional prospective studies with high-quality evidence are still needed to further confirm the causal relationship, relevant mechanisms, and clinical significance.

\section{Acknowledgments}

We greatly acknowledge the American Journal Experts (AJE) and the AME Editing Service (AES) for professional English editing.

Funding: This work was sponsored by the National Natural Science Foundation of China (No. 81970657) and the Shanghai Sailing Program (No. 19YF1432100).

\section{Footnote}

Reporting Checklist: The authors have completed the STROBE reporting checklist. Available at http://dx.doi. org/10.21037/tau-20-914

Data Sharing Statement: Available at http://dx.doi. org/10.21037/tau-20-914

Conflicts of Interest: All authors have completed the ICMJE uniform disclosure form (available at http://dx.doi. org/10.21037/tau-20-914). The authors have no conflicts of interest to declare.

Ethical Statement: The authors are accountable for all aspects of the work in ensuring that questions related to the accuracy or integrity of any part of the work are appropriately investigated and resolved. This study was conducted in accordance with the Declaration of Helsinki (as revised in 2013). The Ethics Committee of Xinhua Hospital Affiliated to Shanghai Jiao Tong University School of Medicine approved the study protocol (XHEC-D-2020-078). All participants provided written informed consent.
Open Access Statement: This is an Open Access article distributed in accordance with the Creative Commons Attribution-NonCommercial-NoDerivs 4.0 International License (CC BY-NC-ND 4.0), which permits the noncommercial replication and distribution of the article with the strict proviso that no changes or edits are made and the original work is properly cited (including links to both the formal publication through the relevant DOI and the license). See: https://creativecommons.org/licenses/by-nc-nd/4.0/.

\section{References}

1. Anderson JB, Roehrborn CG, Schalken JA, et al. The progression of benign prostatic hyperplasia: examining the evidence and determining the risk. Eur Urol 2001;39:390-9.

2. Clifford GM, Logie J, Farmer RDT. How do symptoms indicative of BPH progress in real life practice? The UK experience. Eur Urol 2000;38:48-53.

3. Emberton M, Cornel EB, Bassi PF, et al. Benign prostatic hyperplasia as a progressive disease: a guide to the risk factors and options for medical management. Int J Clin Pract 2008;62:1076-86.

4. Murray K, Massey A, Feneley RC. Acute urinary retention-a urodynamic assessment. Br J Urol 1984;56:468-73.

5. Tubaro A, Carter S, Trucchi A, et al. Early treatment of benign prostatic hyperplasia: implications for reducing the risk of permanent bladder damage. Drugs Aging 2003;20:185-95.

6. Elmissiry MM, Ali AG, Abulfotooh A, et al. Factors determining the amount of residual urine in men with bladder outlet obstruction: Could it be a predictor for bladder contractility? Arab J Urol 2014;12:214-8.

7. Chen JS, Chang CH, Yang WH, et al. Acute urinary retention increases the risk of complications after transurethral resection of the prostate: a population-based study. BJU Int 2012;110:E896-E901.

8. Tyagi P, Motley SS, Koyama T, et al. Molecular correlates in urine for the obesity and prostatic inflammation of BPH/LUTS patients. Prostate 2018;78:17-24.

9. Ozer K, Horsanali MO, Gorgel SN, et al. Association between benign prostatic hyperplasia and neutrophillymphocyte ratio, an indicator of inflammation and metabolic syndrome. Urol Int 2017;98:466-71.

10. Gandaglia G, Briganti A, Gontero P, et al. The role of chronic prostatic inflammation in the pathogenesis and progression of benign prostatic hyperplasia (BPH). BJU 
Int 2013;112:432-41.

11. Gerstenbluth RE, Seftel AD, MacLennan GT, et al. Distribution of chronic prostatitis in radical prostatectomy specimens with up-regulation of bcl-2 in areas of inflammation. J Urol 2002;167:2267-70.

12. Nickel JC, Roehrborn CG, O'Leary MP, et al. The relationship between prostate inflammation and lower urinary tract symptoms: examination of baseline data from the REDUCE trial. Eur Urol 2008;54:1379-84.

13. Nickel JC, Roehrborn CG, O'Leary MP, et al. Examination of the relationship between symptoms of prostatitis and histological inflammation: baseline data from the REDUCE chemoprevention trial. J Urol 2007;178:896-900; discussion 900-1.

14. Mishra VC, Allen DJ, Nicolaou C, et al. Does intraprostatic inflammation have a role in the pathogenesis and progression of benign prostatic hyperplasia? BJU Int 2007;100:327-31.

15. Kramer G, Mitteregger D, Marberger M. Is benign prostatic hyperplasia $(\mathrm{BPH})$ an immune inflammatory disease? Eur Urol 2007;51:1202-16.

16. Sciarra A, Di Silverio F, Salciccia S, et al. Inflammation and chronic prostatic diseases: evidence for a link? Eur Urol 2007;52:964-72.

17. Mustafa G, Ilhan G, Necip P, et al. Do the medical treatment reduces the rate of surgical treatment in suspected cases of chronic prostatitis before prostatectomy? Eur Rev Med Pharmacol Sci 2013;17:119-22.

18. Tuncel A, Uzun B, Eruyar T, et al. Do prostatic infarction, prostatic inflammation and prostate morphology play a role in acute urinary retention? Eur Urol 2005;48:277-83; discussion 283-4.

19. Nickel JC, Roehrborn CG, Castro-Santamaria R, et al. Chronic prostate inflammation is associated with severity and progression of benign prostatic hyperplasia, lower urinary tract symptoms and risk of acute urinary retention. J Urol 2016;196:1493-8.

20. Torkko KC, Wilson RS, Smith EE, et al. Prostate biopsy markers of inflammation are associated with risk of clinical progression of benign prostatic hyperplasia: findings from the MTOPS study. J Urol 2015;194:454-61.

21. Inamura $S$, Ito $H$, Shinagawa $T$, et al. Prostatic stromal inflammation is associated with bladder outlet obstruction in patients with benign prostatic hyperplasia. Prostate 2018;78:743-52.

22. St Sauver JL, Jacobson DJ, McGree ME, et al. Longitudinal association between prostatitis and development of benign prostatic hyperplasia. Urology
2008;71:475-9.

23. Kwon YK, Choe MS, Seo KW, et al. The effect of intraprostatic chronic inflammation on benign prostatic hyperplasia treatment. Korean J Urol 2010;51:266-70.

24. Wu D, Tang HX, Wu Y, et al. The possible association between serum interleukin 8 and acute urinary retention in Chinese patients with benign prostatic hyperplasia. Andrologia 2020;52:e13763.

25. Korrovits P, Poolamets O, Mandar R, et al. Inflammatory reaction found in prostate-specific material - method standardization and proposed optimal cut-off points. Andrology 2017;5:958-63.

26. Nguyen DP, Li J, Tewari AK. Inflammation and prostate cancer: the role of interleukin 6 (IL-6). BJU Int 2014;113:986-92.

27. Shariat SF, Andrews B, Kattan MW, et al. Plasma levels of interleukin-6 and its soluble receptor are associated with prostate cancer progression and metastasis. Urology 2001;58:1008-15.

28. Stark JR, Li H, Kraft P, et al. Circulating prediagnostic interleukin-6 and C-reactive protein and prostate cancer incidence and mortality. Int J Cancer 2009;124:2683-9.

29. Taheri M, Noroozi R, Rakhshan A, et al. IL-6 genomic variants and risk of prostate cancer. Urol J 2019;16:463-8.

30. Green SB. How many subjects does it take to do a regression analysis? Multivariate Behav Res 1991;26:499-510.

31. Chen Q, Liu D, Zhou C, et al. Relationship between critical thinking disposition and research competence among clinical nurses: a cross-sectional study. J Clin Nurs 2020;29:1332-40.

32. Tabachnick B, Fidell L. Using multivariate statistics (6th ed.). Boston, MA: Pearson Education, 2012.

33. Choong S, Emberton M. Acute urinary retention. BJU Int 2000;85:186-201.

34. Chang IH, Oh SY, Kim SC. A possible relationship between testosterone and lower urinary tract symptoms in men. J Urol 2009;182:215-20.

35. Martin SA, Haren MT, Marshall VR, et al. Prevalence and factors associated with uncomplicated storage and voiding lower urinary tract symptoms in community-dwelling Australian men. World J Urol 2011;29:179-84.

36. Xu D, Yu Y, Zhu Y, et al. A new model consists of intravesical prostatic protrusion, prostate volume and serum prostatic-specific antigen in the evaluation of prostate cancer. Pathol Oncol Res 2014;20:439-43.

37. Schenk JM, Kristal AR, Neuhouser ML, et al. Biomarkers of systemic inflammation and risk of incident, symptomatic 
benign prostatic hyperplasia: results from the prostate cancer prevention trial. Am J Epidemiol 2010;171:571-82.

38. Fibbi B, Penna G, Morelli A, et al. Chronic inflammation in the pathogenesis of benign prostatic hyperplasia. Int J Androl 2010;33:475-88.

39. Milonas D, Trumbeckas D. Prostate-specific antigen and transition zone index - powerful predictors for acute urinary retention in men with benign prostatic hyperplasia. Medicina (Kaunas) 2003;39:1071-7.

40. Miyashita H, Kojima M, Miki T. Ultrasonic measurement of bladder weight as a possible predictor of acute urinary retention in men with lower urinary tract symptoms suggestive of benign prostatic hyperplasia. Ultrasound Med Biol 2002;28:985-90.

41. Marberger MJ, Andersen JT, Nickel JC, et al. Prostate volume and serum prostate-specific antigen as predictors of acute urinary retention. Combined experience from

Cite this article as: $\mathrm{Wu} \mathrm{D}$, Shi ZE, Xu D, Wu Y, Qian SB, Qi J. Serum interleukin 6 and acute urinary retention in elderly men with benign prostatic hyperplasia in China: a cross-sectional study. Transl Androl Urol 2021;10(1):455-465. doi: 10.21037/tau20-914 three large multinational placebo-controlled trials. Eur Urol 2000;38:563-8.

42. Bao QB, He GH, Liu GY, et al. Histological changes of the prostate and acute urinary retention in patients with benign prostatic hyperplasia. Zhonghua Nan Ke Xue 2013;19:811-4.

43. Gabay C. Interleukin-6 and chronic inflammation. Arthritis Res Ther 2006;8 Suppl 2:S3.

44. Korrovits P, Punab M, Turk S, et al. Seminal microflora in asymptomatic inflammatory (NIH IV category) prostatitis. Eur Urol 2006;50:1338-44; discussion 1344-6.

45. Penna G, Mondaini N, Amuchastegui S, et al. Seminal plasma cytokines and chemokines in prostate inflammation: interleukin 8 as a predictive biomarker in chronic prostatitis/chronic pelvic pain syndrome and benign prostatic hyperplasia. Eur Urol 2007;51:524-33. 